\title{
Observation of High Impurity Ion Temperatures in ECW/EBW Sustained Plasmas on LATE
}

\author{
Akira EJIRI, Hitoshi TANAKA ${ }^{1)}$, Wataru TAKAHASHI, Akito SATO, Yuichi TAKASE, \\ Naoto TSUJII, Masaki UCHIDA ${ }^{1)}$ and Takashi MAEKAWA ${ }^{1)}$ \\ Graduate School of Frontier Sciences, The University of Tokyo, Kashiwa 277-8561, Japan \\ ${ }^{1)}$ Graduate School of Energy Science, Kyoto University, Kyoto 606-8502, Japan
}

(Received 10 August 2017 / Accepted 5 September 2017)

\begin{abstract}
Carbon and oxygen impurity line emissions from LATE spherical tokamak plasmas were measured using a visible spectrometer. A plasma current of approximately $10 \mathrm{kA}$ was sustained by ECW/EBW with a frequency of $5 \mathrm{GHz}$ and a power of $130 \mathrm{~kW}$. Emissions of CIII $(464.7 \mathrm{~nm}), \mathrm{OV}(278.1 \mathrm{~nm})$ and CV $(227.1 \mathrm{~nm})$ were observed, and the ion temperatures calculated from the Doppler broadening reached 40 \pm 8, $110 \pm 10$ and $130 \pm 30 \mathrm{eV}$, respectively. The high ion temperatures cannot be explained by collisional heating from electrons, suggesting that the ions are directly heated via unidentified mechanisms.
\end{abstract}

(c) 2017 The Japan Society of Plasma Science and Nuclear Fusion Research

Keywords: electron cyclotron wave, electron Bernstein wave, current drive, spherical tokamak, ion temperature

DOI: $10.1585 /$ pfr.12.1202041

In order to realize a compact spherical tokamak (ST) reactor, the elimination of the massive central solenoid is necessary. RF waves such as electron cyclotron waves and lower hybrid waves are often used to start-up and sustain ST plasmas [1,2]. It is believed that these RF sustained plasmas comprise high energy electrons which carry the plasma current and dominate equilibrium [3,4], low temperature bulk electrons $[5,6]$ and very low temperature ions. In order to understand the power flow in these RF sustained plasmas, the measurement of each component's temperature is crucial.

The ion temperatures in LATE electron cyclotron wave (ECW: $2.45 \mathrm{GHz} / 50 \mathrm{~kW}$ ) sustained plasmas and TST-2 lower hybrid wave (LHW: $200 \mathrm{MHz} / 20 \mathrm{~kW}$ ) sustained plasmas were about 10 and $4 \mathrm{eV}$, respectively [7]. Although the bulk electron temperature of LATE plasmas was not measured, it was probably similar to those in TST-2 (a few tens of electron volts), because both showed similar visible spectra, where CIII $\left(464.7 \mathrm{~nm}, \mathrm{C}^{2+}\right.$, $\left.E_{\mathrm{i}}=47.9 \mathrm{eV}\right)$ intensity was strong and $\mathrm{OV}\left(278.1 \mathrm{~nm}, \mathrm{O}^{4+}\right.$, $\left.E_{\mathrm{i}}=114 \mathrm{eV}\right)$ and $\mathrm{CV}\left(227.1 \mathrm{~nm}, \mathrm{C}^{4+}, E_{\mathrm{i}}=392 \mathrm{eV}\right)$ were too weak to be identified. Here $E_{\mathrm{i}}$ denotes the ionization energy of each element. In this study, the ion temperatures of LATE plasmas sustained by ECW and/or electron Bernstein wave (EBW) with a frequency of $5 \mathrm{GHz}$ and a power of up to $130 \mathrm{~kW}$ are presented.

Figure 1 shows the time evolution of a typical discharge. CIII, OV and CV line intensities and the ion temperatures measured by the same visible spectrometer used in our precious study [7] are displayed. The intensities are appropriately scaled to be visible on the same graph. The
CIII emission is present from the beginning of a discharge, while the intensity of OV emission grows until $t \sim 90 \mathrm{~ms}$; further, it rapidly increases. The $\mathrm{CV}$ emission becomes visible at $t \sim 100 \mathrm{~ms}$. Considering the detector (photomultiplier tube) gain variation due to the applied high voltage, the signal intensity ratio between $\mathrm{OV}$, CIII and CV was $25: 5: 1$ at $t \sim 110 \mathrm{~ms}$. These time evolutions of OV, CIII and $\mathrm{CV}$ emissions suggest a monotonically increasing bulk
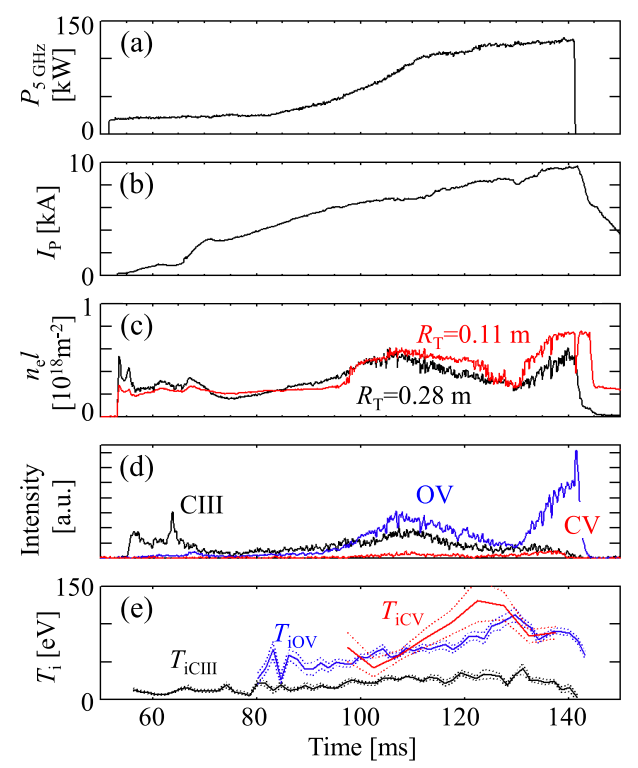

Fig. 1 Waveforms of injected ECW power $P_{5 \mathrm{GHz}}$ (a), plasma current $I_{\mathrm{P}}(\mathrm{b})$, line integrated density $n_{\mathrm{e}} l$ with tangency radii $R_{\mathrm{T}}=0.11,0.28 \mathrm{~m}(\mathrm{c}), \mathrm{CIII}, \mathrm{OV}, \mathrm{CV}$ intensities (d) and ion temperatures measured at $R_{\mathrm{T}}=0.21 \mathrm{~m}(\mathrm{e})$. 
electron temperature. In a TST-2 Ohmic discharge, CV appears when $T_{\mathrm{e}} \sim 150 \mathrm{eV}$. The bulk electron temperature in LATE was probably similar to this temperature.

Figure 1 (e) shows ion temperatures for CIII, OV and $\mathrm{CV}$, and each of these tends to increase with time. The maximum temperatures during the discharge are $40 \pm 8$, $110 \pm 10$ and $130 \pm 30 \mathrm{eV}$, respectively. Here, the error bars represent the fitting errors, which mainly originate from the random noise in weak signals. Thus, we need a long accumulation time for the weak CV signals. The temperature of CIII is the lowest among them, and those of OV and $\mathrm{CV}$ are higher. This is probably due to the spatial profile of ion temperature profile, because the emission profile for CIII is broad while that for OV is more peaked according to multiple line of sight measurements. These results imply that the bulk electron and ion temperatures have peaked profiles and increase with time.

Here, we assume collisional ion heating by electrons, and calculate the time evolution of ion temperature for a given assumed time evolution of bulk electron temperature to evaluate the contribution of collisional heating. The equation for ion temperature is given as follows:

$$
\frac{\mathrm{d}}{\mathrm{d} t}\left(\frac{3}{2} n_{\mathrm{i}} T_{\mathrm{ic}}\right)=\frac{3}{2} n_{\mathrm{i}} \frac{T_{\mathrm{eg}}-T_{\mathrm{ic}}}{\tau_{\mathrm{ei}}}-\frac{3}{2} \frac{n_{\mathrm{i}} T_{\mathrm{ic}}}{\tau_{\mathrm{E}}},
$$

where $T_{\text {ic }}$ is the calculated ion temperature, $T_{\text {eg }}$ is a given time-dependent electron temperature, $n_{\mathrm{i}}$ is the ion density and $\tau_{\mathrm{E}}$ is the ion energy confinement time. $\tau_{\mathrm{ei}}$ is the electron-ion temperature relaxation time given by [8]

$$
\tau_{\text {ei }}=1.58 \times 10^{-7}[\mathrm{~s}] \frac{A T_{\mathrm{e}}[\mathrm{eV}]^{3 / 2}}{Z^{2} n_{\mathrm{e}}\left[10^{20} \mathrm{~m}^{-3}\right]},
$$

where $A(=1)$ and $Z(=1)$ are the mass number and charge number of hydrogen, respectively. An example of relaxation time between electrons and hydrogen ions is $30 \mathrm{~ms}$ for $T_{\mathrm{e}}=100 \mathrm{eV}, n_{\mathrm{e}}\left(=n_{\mathrm{i}}\right)=6 \times 10^{17} \mathrm{~m}^{-3}$, while that between hydrogen and the impurities is about $3 \mathrm{~ms}$ for $T_{\mathrm{i}}=100 \mathrm{eV}$. We make several assumptions to obtain the upper limit of the achievable ion temperature. First, we adopt a zero-dimensional model, neglecting spatial variations, and we use measured or estimated central values to calculate Eq. (1). In addition, we neglect the density evolution, then the equation for the ion temperature can be rewritten as follows:

$$
\frac{\mathrm{d} T_{\mathrm{ic}}}{\mathrm{d} t}=\frac{T_{\mathrm{eg}}-T_{\mathrm{ic}}}{\tau_{\mathrm{ei}}}-\frac{T_{\mathrm{ic}}}{\tau_{\mathrm{E}}} .
$$

Second, we neglect the temperature relaxations between ions. Third, we adopt ITER-89P energy confinement time [9] for $\tau_{E}$ in Eq. (3), but we multiply it by a factor much larger than one to obtain the upper limit. The major and minor radii and the line averaged electron density, which are necessary to calculate $\tau_{\mathrm{E}}$, are estimated from the line integrated densities measured at $R_{\mathrm{T}}=0.11,0.19$ and $0.28 \mathrm{~m}$, assuming a parabolic density profile. For $n_{\mathrm{e}}$ in the denominator of Eq. (2), we use the estimated central density. Fig-

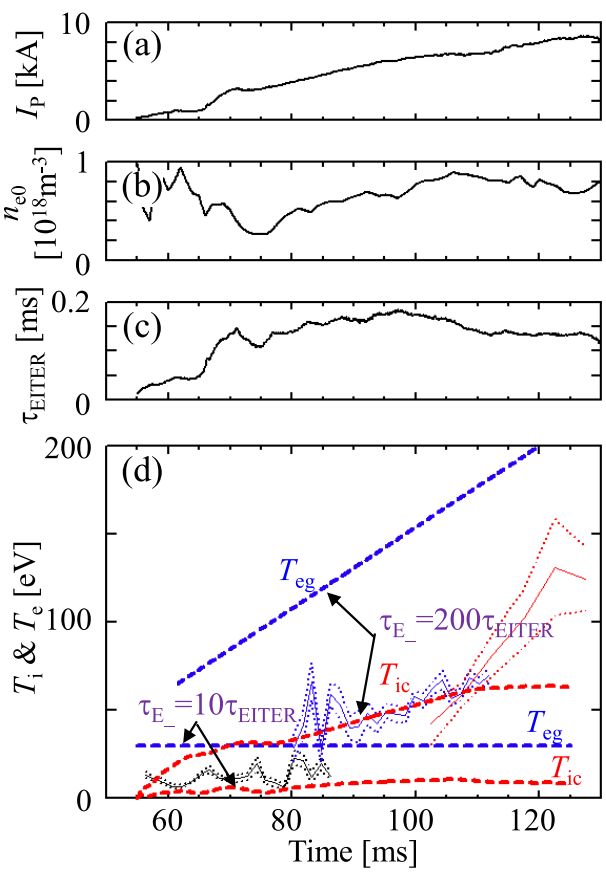

Fig. 2 Time evolutions of $I_{\mathrm{P}}(\mathrm{a}), n_{\mathrm{e} 0}(\mathrm{~b}), \tau_{\text {EITER }}(\mathrm{c})$ and $T_{\text {eg }}, T_{\text {ic }}$ (d). In (d) two cases: $\tau_{\mathrm{E}}=10 \times, 200 \times \tau_{\text {EITER }}$ are shown. The thin curves in (d) indicate the measured CIII, OV and CV temperatures shown in Fig. 1 (e).

ures 2 (b) and (c) show the estimated central electron density $n_{\mathrm{e} 0}$ and the calculated energy confinement time $\tau_{\text {EITER }}$. In order to obtain the upper limit of the ion temperature, $T_{\text {eg }}$ should be sufficiently high to heat ions, but it should not be too high because of the following reason. When $T_{\text {eg }} \gg T_{\text {ic }}$, the ion heating term $\left(T_{\text {eg }}-T_{\text {ic }}\right) / \tau_{\text {ei }}$ is approximated to $T_{\mathrm{eg}} / \tau_{\mathrm{ei}}$, and it is proportional to $1 / \sqrt{T_{\mathrm{eg}}}$ owning to the $T_{\mathrm{e}}^{3 / 2}$ dependence in Eq. (2). Thus, there is an optimum $T_{\text {eg }}$ to heat ions efficiently.

We tried various time evolution of $T_{\text {eg }}$ via scanning the initial temperature $T_{\text {eg }}(55)$ (at $t=55 \mathrm{~ms}$ ) and temperature increment or decrement $\Delta T_{\text {eg }}$ as follows:

$$
T_{\text {eg }}(t)=T_{\text {eg }}(55)+\Delta T_{\text {eg }}(t-55)^{\alpha},
$$

where $\alpha=0,0.5$, and 1 . Figure 2(d) shows the highest ion temperatures for the two cases: $\tau_{\mathrm{E}}=10 \tau_{\mathrm{EITER}}, 200 \tau_{\mathrm{EITER}}$. For the former case, a constant $T_{\text {eg }}=30 \mathrm{eV}$ is optimum in the sense that it yields the highest ion temperature of $11 \mathrm{eV}$. This indicates that the observed high ion temperature above $100 \mathrm{eV}$ cannot be explained by collisional heating from electrons even if we reduced the energy loss by adopting $\tau_{\mathrm{E}}=10 \tau_{\text {EITER }}$. The case with $\tau_{\mathrm{E}}=200 \tau_{\mathrm{EITER}}$ is an extreme case, which still cannot explain the high ion temperature.

The observed ion temperatures of $\sim 100 \mathrm{eV}$ are much higher than the two cases reported in [7]. Besides these cases, the ion temperatures in TST-2@K ECH $(8.2 \mathrm{GHz})$ [10], QUEST ECH (8.2 GHz)[11] and QUEST ECH $(28 \mathrm{GHz})[12]$ started-up plasmas are less than or around 
$10 \mathrm{eV}$. Therefore, the present high ion temperature is the highest among those in RF started-up ST plasmas. Although the present high ion temperature is reproducible in the same operational conditions, we have not yet clarified the necessary conditions, and further studies are required. Here, we list three cases in Ohmically (i.e., inductively) started-up ST plasmas indicating direct ion heating. In MAST and TST-2, abrupt ion temperature increases were observed during MHD events and the heating is probably due to magnetic reconnection $[13,14]$. In NSTX, during high harmonic fast wave injection, a high edge ion temperature was observed, and it was suggested that an ion Bernstein wave generated by the parametric decay of the injected wave heats the ions [15].

In conclusion, impurity line emissions of CIII, OV and $\mathrm{CV}$ from the LATE plasmas sustained by ECW/EBW were measured using a visible spectrometer. The OV signal showed the strongest intensity among them, and the maximum impurity ion temperature exceeded $100 \mathrm{eV}$. This ion temperature is much higher than that expected from the collisional heating, suggesting some unidentified direct ion heating mechanisms.
This work is supported by JSPS Grant-in-Aid for Scientific Research (S) (21226021), by NIFS Collaboration Research Program NIFS14KOCR001 and NIFS16KNWR002, and by JSPS A3 Foresight Program.

[1] C.B. Forest et al., Phys. Rev. Lett. 68, 3559 (1992).

[2] Y. Takase et al., Nucl. Fusion 51, 063017 (2011).

[3] M. Uchida et al., Phys. Rev. Lett. 104, 065001 (2010).

[4] K. Mishra et al., Nucl. Fusion 55, 083009 (2015).

[5] T. Yamaguchi et al., Plasma Fusion Res. 8, 1302001 (2013).

[6] H. Togashi et al., Plasma Fusion Res. 10, 1202082 (2015).

[7] S. Tsuda et al., Plasma Fusion Res. 10, 1202064 (2015).

[8] e.g. K. Miyamoto, Fundamentals of Plasma Physics and Controlled Fusion (Iwanami Book Service Center, Tokyo, 1997).

[9] P.N. Yushmanov et al., Nucl. Fusion 30, 1999 (1990).

[10] A. Ejiri et al., Nucl. Fusion 46, 709 (2006).

[11] T. Shikama et al., to be submitted.

[12] T. Shikama, private communication.

[13] A. Sykes, Plasma Phys. Control. Fusion 43, A127 (2001).

[14] A. Ejiri et al., Nucl. Fusion 43, 547 (2003).

[15] T.M. Biewer et al., Phys. Plasmas 12, 056108 (2005). 